\title{
An Extranasopharyngeal Angiofibroma of Mandibular Ramus in a Preschool Child - An Extremely Rare Case Report
}

\author{
Charan Teja Vemagiri ${ }^{1}$, Chandrababu Pamidi², Srikanth Damera ${ }^{3}$, Supraja Naga Atluri ${ }^{4}$, Mounika Kallukuri ${ }^{5}$ \\ 1,4,5 Department of Paediatric and Preventive Dentistry, GSL Dental College and Hospital, \\ Rajahmahendravaram, Andhra Pradesh, India, 2, 3 Department of Oral and Maxillofacial Surgery, GSL \\ Dental College and Hospital, Rajahmahendravaram, Andhra Pradesh, India.
}

\section{INTRODUCTION}

Angiofibroma or juvenile nasopharyngeal angiofibroma (JNF) is a rare vascular benign tumour predominant in male adolescents and pre-adolescents. In spite of several origin sites reported, nasopharynx in the region of the sphenopalatine foramen and pterygopalatine fossa remains most common. ${ }^{1}$ Nevertheless, sporadic description of extra nasopharyngeal angiofibromas (ENAF) are also rarely evident. ${ }^{2}$ Literature documents maxillary sinus as the most favourable site for ENAF followed by the ethmoid sinus, nasal cavity, nasal septum, larynx, sphenoid sinus, cheek, conjunctiva, oropharynx, retromolar area and others. ${ }^{3}$ However, ENAF of mandibular ramus marks rarity and no case has been reported with respect to this anatomic location especially in preschool children yet. Hence, a rare ENAF in a 3-year-old child, principally confined to mandible, with neither sphenopalatine foramen nor nasopharynx involvement presenting with swelling is described

\section{PRESENTATION OF CASE}

A three-year-old male patient reported with a 3-month history of non-painful swelling in the left mandibular ramus region. Trauma with immediate pain history before 3 months was evident. A small swelling was observed around 15 days later by his parents, which gradually grew to present size. Despite taking medications prescribed by the local clinician, swelling did not subside. His past medical, surgical and dental history was non-contributory.

Extraoral examination revealed a swelling measuring $2.5 \mathrm{~cm} \times 2.5 \mathrm{~cm}$ in the mandibular ramus extending superoinferiorly from $1 \mathrm{~cm}$ short of both upper to lower border of ramus and anteroposteriorly from canine mesial surface to first molar distal surface. Upon palpation, swelling was mildly warm, non-tender with variable consistency, firm to hard in centre and soft to firm at the periphery with normal overlying skin. Left submandibular lymph nodes were non palpable. Intraorally, swelling with vestibular tenderness was noted in the 73 - 74 regions with normal overlying mucosa.
Corresponding Author: Dr. Charan Teja Vemagiri, Department of Paediatric and Preventive Dentistry, GSL Dental College and Hospital, Rajahmahendravaram, Andhra Pradesh, India. E-mail: charantejavemagiri@gmail.com

DOI: $10.14260 /$ jemds $/ 2021 / 434$

How to Cite This Article:

Vemagiri CT, Pamidi C, Damera S, et al. An extranasopharyngeal angiofibroma of mandibular ramus in a preschool child - an extremely rare case report. J Evolution Med Dent Sci 2021;10(28):2128-2130, DOI: $10.14260 /$ jemds $/ 2021 / 434$

Submission 22-03-2021, Peer Review 14-05-2021, Acceptance 18-05-2021, Published 12-07-2021.

Copyright (C) 2021 Charan Teja Vemagiri et al. This is an open access article distributed under Creative Commons Attribution License [Attribution 4.0 International (CC BY 4.0)] 
Patient's young age hindered in obtaining pre-operative radiographs. Routine haematological investigations gave normal values. Fine-needle aspiration cytology showed clusters of spindle cells in a background of psammoma bodies and osteoid material with no nuclear atypia and malignant cells suggestive of benign odontogenic tumour. After obtaining the parent's consent, diagnostic and therapeutic excisional biopsy was performed under local anaesthesia through intraoral incision. (Figure 1 and 2) Suturing was done and the patient was kept on antibiotic and analgesics.
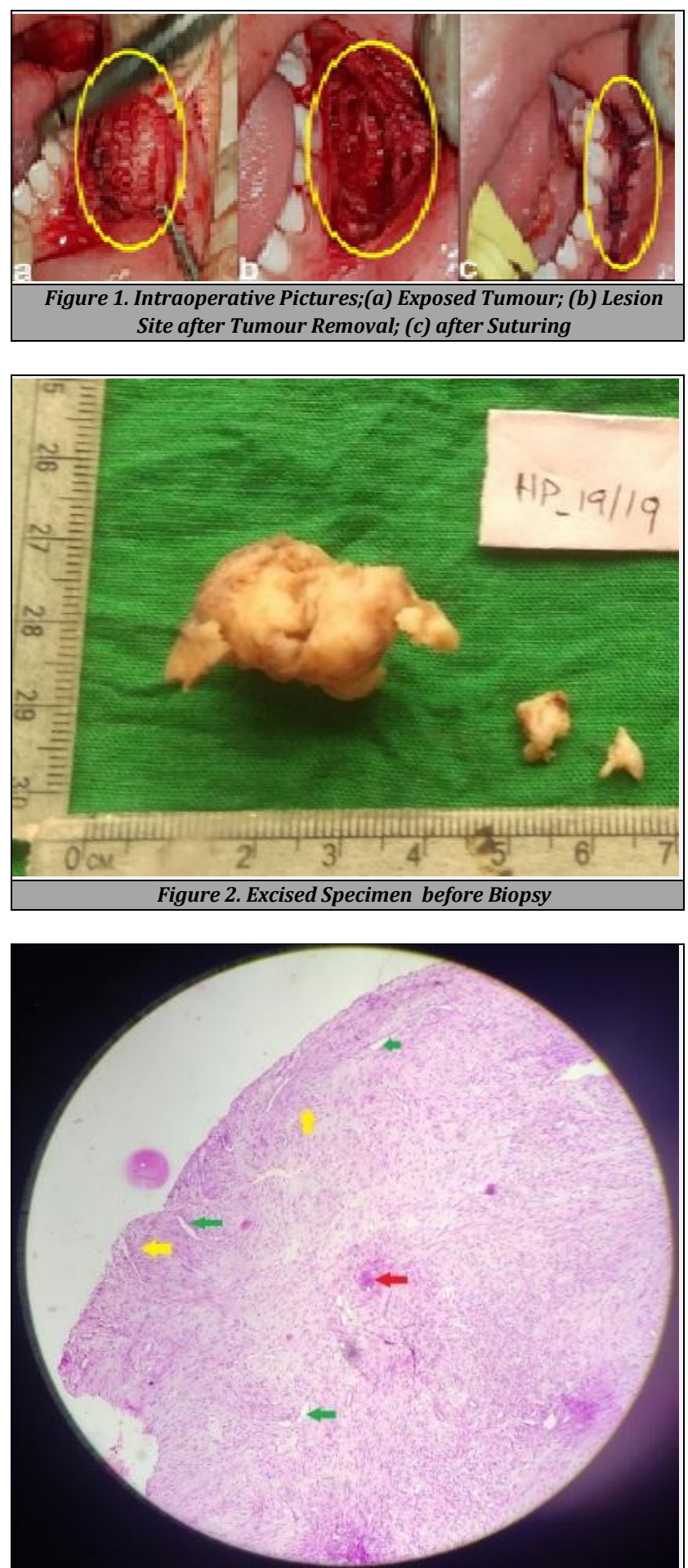

Figure 3. Histopathology Showing Fibrous Connective Tissue Stroma with Spindle Cells (Yellow Arrows), Dilated Blood Vessels (Green Arrows), Hyalinised Areas (Red Arrows)
Microscopic examination showed fibrous connective tissue stroma with partial encapsulation exhibiting numerous spindle shaped cells arranged in fascicles and focal areas with cross-section of nerve bundles. Numerous dilated capillaries lined by endothelial cells and some hyalinised areas were also observed.

These findings were found to be compatible with angiofibroma. (Figure 3) In addition, immunohistochemistry marker S-100 was negative which underpins the angiofibroma diagnosis. (Figure 4)
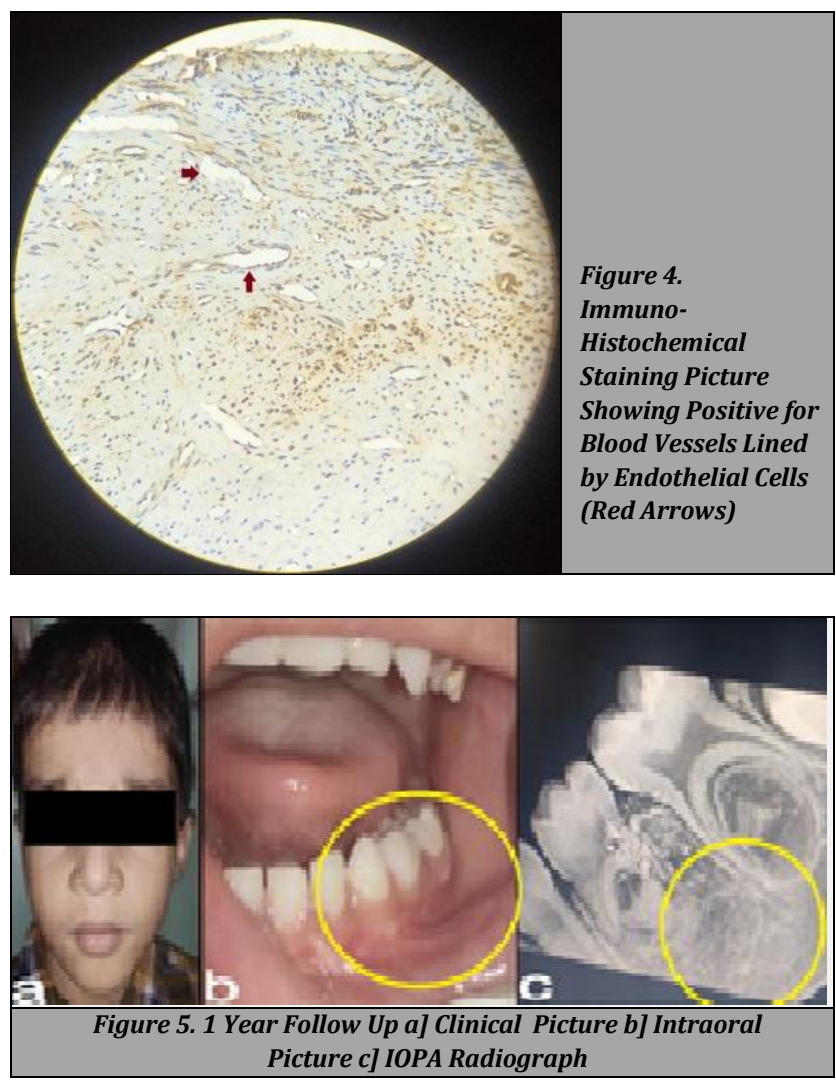

After a follow up for 1-year, uneventful post-operative wound healing with no recurrence was observed. (Figure 5)

\section{DISCUSSION}

Current literature shows evidence that angiofibromas originating outside the nasopharynx i.e., vicinity of sphenopalatine foramen and pterygoid plates are termed extra nasopharyngeal angiofibromas (ENAF), and around 65 ENAF's with maxillary sinus as most common site were reviewed by Windfuhr JP and Remmert $\mathrm{S}$. Though, numerous ENAF sites were published, ENAFs involving buccal space are rarely reported.2,4 Furthermore, significance of reporting this case lies in its involvement of mandibular ramus, as no published documentation involving this anatomic location especially in preschool children is present till date.

Despite proposal of congenital, genetic, hormonal and vascular theories, origin and pathogenesis of both JNFs and ENAFs is still disputed. ${ }^{5}$ Moreover, ENAFs differ from JNFs in terms of location, symptoms, age, gender and clinical features accounting for tag atypical angiofibroma..$^{3,4}$ Nevertheless, this will not suffice in all patients to distinguish between them. ${ }^{2}$ 
Literature documents that nasopharyngeal angiofibromas are common in 12-14-year-old males while ENAFs between 17 and 22 year females. ${ }^{6}$ Contrary to this, the present ENAF was reported in the mandibular ramus of a 3-year preschool child confirming its rarity.

Depending on the location, ENAFs present variable symptoms resulting in more confusing clinical presentation and the delay between the onset of symptoms and the diagnosis. ${ }^{7}$ In our case, with the tumour arising from ramus of mandible, the only symptom was minor dysphagia.

Even though benign histopathologically, JNFs and ENAFs may grow significantly eroding bone and invading regional structures causing structural and functional damage. However, no clear explanation is documented regarding this aggressiveness of JNFs and ENAFs. ${ }^{8}$ Likewise, FNAC of present case suggested the benign nature of the tumour with no nuclear atypia and absence of malignant cells. Nevertheless, erosion of the ramal bone was also observed. ENAFs simulate JNFs histologically and characterised by rich vascular network typically lacking muscular elastic laminae within a fibrous stroma resulting in massive bleeding following biopsies and excision. ${ }^{4}$ However, it was detected only in half of the patients with ENAFs in whom biopsy was performed as ENAFs constitute a more heterogeneous group and the blood supply to lesion depends on its point of origin and location. ${ }^{2,7}$ Supporting the above statement, no excessive bleeding was observed in our patient also after excisional biopsy.

As for JNFs, surgical resection for limited ENAFs and radiotherapy for extensive unresectable lesions is the preferred treatment of choice. However, surgical approach is tailored based on experience, skill of surgeon, location and size of tumor.2,3,5,9 Taking the benign nature and size of tumour into consideration, total resection of lesion was done under local anaesthesia in the present patient for both therapeutic and diagnostic purposes.

Though angiofibroma can be diagnosed on clinical and computed tomography characteristics, histopathological confirmation is essential.3,5,6 The excised specimen of present case also revealed the characteristic features of angiofibroma and was further confirmed by immunohistochemical (IHC) findings which showed negativity for desmin and revealed positive for vimentin and alpha actin. ${ }^{10}$

Recurrence rate of JNFs range from $0-57 \%$ following treatment and it depends on the amount of lesion excised, vascularity, and age of onset, site, and extension of lesion. 2,5 Unlike this, recurrence was not reported for patients with ENAFs as extrapharyngeal location eases total resection of lesion. ${ }^{6}$ In agreement with documented evidence, no recurrence was observed even after 1 year in the present case as well.

\section{CONCLUSIONS}

ENAF within the mandible is an extremely rare tumour. Different pathogenesis, epidemiology, variable signs and symptoms present a diagnostic challenge in differentiating it from NA. However, it must be taken into consideration in the differential diagnosis of vascular tumours of head and neck suggestive of a separate entity.

Financial or other competing interests: None.

Disclosure forms provided by the authors are available with the full text of this article at jemds.com.

\section{REFERENCES}

[1] Singh R, Hazarika P, Nayak DR, et al. Role of Le Fort type I osteotomy approach in juvenile nasopharyngeal angiofibroma. Int J Oral Maxillofac Surg 2011;40(11):1271-4

[2] Windfuhr JP, Remmert S. Extranasopharyngeal angiofibroma: etiology, incidence and management. Acta Otolaryngol 2004;124(8):880-9.

[3] Goud S, Swapna T, Bindu H, et al. Extra nasopharyngeal angiofibroma simulating a mucocele: a new location for the rare entity. J Clin Diagn Res 2017;11(1):ZD28-30.

[4] Nandhini J, Ramasamy S, Kaul RN, et al. Juvenile primary extranasopharyngeal angiofibroma, presenting as cheek swelling. J Oral Maxillofac Pathol 2018;22(Suppl 1):S736.

[5] Pardhe N, Chhibber N, Agarwal D, et al. Juvenile nasopharyngeal angiofibroma extending into the oral cavity: a rare entity. J Clin Diagn Res 2015;9(6):ZD31-3.

[6] De Barros Baptista MA, De Rezende P, Voegels RL. Extranasopharyngeal angiofibroma originating in the inferior turbinate: a distinct clinical entity at an unusual site. Int Arch Otorhinolaryngol 2014;18(4):403-5.

[7] Szymanska A, Szymanski M, Morshed K, et al. Extranasopharyngeal angiofibroma: clinical and radiological presentation. Eur Arch Otorhinolaryngol 2013;270(2):655-60.

[8] Khaliq MIU, Shah AA, Dar N. A rare case of angiofibroma of the mandible: a case report. J Oral Biol Craniofac Res 2016;6(2):168-70.

[9] Gupta N, Dass A, Saini V, et al. Extranasopharyngeal angiofibroma: a diagnostic dilemma. Philipp J Otolaryngol Head Neck Surg 2018;33(1):39-42.

[10] Peric A, Sotirovic J, Cerovic S, et al. Immunohistochemistry in diagnosis of extranasopharyngeal angiofibroma originating from nasal cavity: case presentation and review of literature. Acta Medica (Hradec Kralove) 2013;56(4):133-41. 